\title{
Anomalous force diffusion in nearly-ordered packings of frictionless discs
}

\author{
D.A. Head $\dagger$ \\ $\dagger$ Department of Physics and Astronomy, Vrije Universiteit $1081 \mathrm{HV}$, Amsterdam, The Netherlands
}

\begin{abstract}
We derive analytic expressions for force propagation in packings of frictionless discs with a narrow distribution of disc sizes, by expanding to first order about the known ordered solution. The distribution of contact forces $P(f)$ is found to be narrow at the upper surface, and broaden at a rate that varies with depth, being superdiffusive near the surface until crossing over to a subdiffusive regime near the fixed base. Furthermore, the response to an isolated load propagates along the edge of a 'cone,' as in the ordered case, but fluctuates under ensemble averaging by an amount that depends purely on height, not on the lateral position. Finally, we comment on ways in which the analytical framework presented here could be extended to a wider range of granular packings.
\end{abstract}

45.70.Cc, 05.40.-a, 83.80.Fg

\section{INTRODUCTION}

The mechanical properties of static bead packings remains a controversial and largely unanswered problem of granular media [1-4]. Experiments and numerical simulations have thus far been unable to provide a definitive description of the propagation of stresses through rigid bead packings, in part because of the limited range of bead properties and system sizes typically considered. Theory is not yet at a sufficiently advanced stage to resolve these deficiencies. First-principle approaches such as those initiated in [5-8] may eventually generate some form of 'complete' solution, but their significant complexity has thus far ruled out easily verifiable predictions. Analytically tractable theories have been devised, such as the $q$-model [9-11] and the force ray splitting 'double Y' model $[3,12,13]$, but only after making ad hoc if intuitive assumptions regarding the local propagation of forces.

An alternative approach to treating granular piles within an analytical framework is to restrict attention to a specific class of packings, but otherwise treat it as exactly as possible. Frictionless spheres are an ideal test case: the smooth surfaces cannot support tangential contact forces, removing indeterminacy problems that arise with frictional surfaces, and furthermore the spherical nature of the beads means that the remaining normal component must pass through the bead centre, so that the torque applied at each contact must vanish. Nonetheless disordered frictionless sphere packings are far from trivial: apart from mean field-type analysis [14], results obtained thus far have typically been numerical, either for small systems with arbitrary disorder $[15,16]$, or much larger systems within the 'approximation of small displacements' of Roux et al. $[17,18]$ (in this context, it is perhaps also relevant to mention the large-scale simulations of Breton et al. on geometrically ordered packings with disorder in the contact friction [19]).

For a completely ordered lattice of frictionless discs, all of the forces propagate along the directions of colinear bonds, and it is straightforward to write down all of the contact forces in response to a specified loading. In this paper, we expand about this ordered solution to first order in the deviations of bead radii from their mean value, and derive analytical expressions for the propagation and distribution of forces for piles of arbitrary size. This we are able to eliminate statistical noise. Our findings are fully detailed in the subsequent sections, but in brief: the distribution of contact forces $P(f)$ is narrow near the surface, and broadens as one moves further down the pile. This broadening is initially superdiffusive (i.e. faster than the square root of distance from the surface), but crosses over to a subdiffusive regime lower down the pile and ceases to broaden precisely at the base. Turning to consider the Green's function problem of the response in contact forces to an isolated point load, we find that mean of this response is concentrated along a 'cone' extending downwards from the point source along lines of contacts. Although the response inside the cone averages to zero, the variance in the fluctuations under ensemble averaging can be calculated, and is found to depend purely on the height, not (as might be expected) the distance from edge of the cone.

This paper is arranged as follows. In Sec. II we specify in detail the system to be considered. The statistical properties of the geometry of the packing under the specified construction procedure and boundary conditions are derived in Sec. III. The force propagation from a single loaded bead within a particular packing is detailed in Sec. IV, which is then averaged under two types of loading to give the distribution of contact forces $P(f)$ in Sec. V. In Sec. VI the response to an isolated load is found, including its statistical properties under ensemble averaging. Finally, in Sec. VII we summarise and suggest possible ways in which the analytical framework presented here might be extended. We remark that our approach is similar in spirit to Roux et al.'s 'assumption of small displacements,' although here were consider low-density packings for which the disorder cannot make or break contacts. In fact, it is essentially the small-polydispersity of the adaptive network algorithm of Tkachenko and Witten [16], treated analytically rather than numerically. 


\section{PRELIMINARIES}

Here we specify the class of granular packings under consideration in this paper, as well as the reasons for their analytically tractability. Firstly, we consider packings that are marginally rigid or isostatic [14,20], i.e. the number of (scalar) constraints imposed by the conditions of force and torque balance on each bead equals the number of degrees of freedom in the contact forces. Then the force network can be determined purely from the requirements of local force and torque balance, without reference to the deformation properties of the beads. Also, since both the force and torque balance equations are linear, the full contact network can be found by simply summing the forces propagating from each loaded bead.

Secondly, our packings are constructed from frictionless beads, so that only the normal components of the contact forces are non-zero. This means that the direction of the contact forces can be uniquely determined from the geometry. Furthermore we use spherical beads, so that these contact forces point through the bead centres and all torques trivially vanish. It is straightforward to calculate the isostatic limit for such packings. If the mean number of contacts per bead is $Z$, this represent $Z / 2$ degrees of freedom in the (normal) contact forces, and $d$ constraints imposed by force balance in a $d$-dimensional system. Thus the isostatic limit for frictionless spheres has $Z=2 d$.

Thirdly, we suppose that the beads are almost monodisperse in size, i.e. the bead radii are narrowly distributed about some mean value. Then the contact topology will be the same as for a completely ordered system. Since $Z=2 d$ everywhere, the load on any one bead can be uniquely decomposed into the $d$ contact forces of the two lower beads (assuming there are no horizontal contacts). In this manner the forces due to external loads will propagate downwards through the system until reaching the fixed base $[16,21]$.

Finally, the packing is formed by the sequential deposition of beads to the upper surface, and ensuring mechanical stability between each addition. This exploits a happy coincidence of numbers: for $Z=2 d$ packing, each incoming bead will rest on $d$ others, which is also the number of contacts required to determine the position of the added bead. Thus the geometry of the pile can be found without needing to know the incoming bead trajectories.

\section{GEOMETRY OF THE PACKING}

In this section, the position vectors of the beads for a particular set of bead radii is derived for the $d=2$ case of frictionless discs. An assumption underlying the analysis throughout this paper is that all of the contact forces are compressive. This is crucial, since granular particles are typically regarded as non-cohesive and therefore cannot support tensile contacts. It is clear that such a state must exist if the disorder is sufficiently small, since all contact forces are compressive in the corresponding ordered limit [22]. The range of validity of this assumption will be discussed in Sec. V after the force distribution has been found.

Before considering the near-crystalline packing, it is useful to describe the completely ordered case, both as reference and to fix the notation. See Fig. 1. Without disorder, each bead has radius $r$ and is located at a lattice site $(i, j)$, where $i$ and $j$ refer to the horizontal and vertically-upwards lattice directions, respectively. The centre of the bead at $(i, j)$ is denoted by the position vector $\mathbf{x}_{i j}$, where

$$
\mathbf{x}_{i j}=r(j+i) \hat{\mathbf{n}}^{+}+r(j-i) \hat{\mathbf{n}}^{-}
$$

Here, $\hat{\mathbf{n}}^{ \pm}$are the unit base lattice vectors in the upwardsright $\left(\hat{\mathbf{n}}^{+}\right)$and upwards-left $\left(\hat{\mathbf{n}}^{-}\right)$directions. It is straightforward to show that $\hat{\mathbf{n}}^{ \pm}=\left( \pm n_{x}, n_{y}\right)$ with $n_{x}=s / 4 r$, where $s$ is the horizontal distance between bead centres. Each bead makes $2 d=4$ contacts with beads aligned along the lattice diagonals, i.e. bead $(i, j)$ touches beads $(i \pm 1, j \pm 1)$. Thus only sites with $i+j$ even are occupied. The requirement that beads cannot overlap in either the horizontal or vertical directions fixes $s$ to be in the range $(2 r, 2 \sqrt{3} r)$.

Disorder is introduced into the packing by assigning each bead a radius $r+\delta r_{i j}$ prior to deposition onto lattice site $(i, j)$, where the $\delta r_{i j}$ are random variables drawn from a distribution with zero mean and variance $\sigma_{\delta r}^{2}$. When a bead is added to the growing surface, its centre $\mathbf{x}_{i j}+\delta \mathbf{x}_{i j}$ must be chosen to ensure the distance to both supporting beads is equal to the sum of the corresponding radii; to first order in the $\delta r_{i j}$, this gives

$$
\begin{aligned}
\delta \mathbf{x}_{i j}= & \frac{1}{2}\left(\delta \mathbf{x}_{i-1 j-1}+\delta \mathbf{x}_{i+1 j-1}\right) \\
& -\frac{1}{2}\left(\begin{array}{cc}
0 & \frac{n_{y}}{n_{x}} \\
\frac{n_{x}}{n_{y}} & 0
\end{array}\right)\left(\delta \mathbf{x}_{i+1 j-1}-\delta \mathbf{x}_{i-1 j-1}\right) \\
& +\left(\delta r_{i j}+\delta r_{i-1, j-1}\right) \mathbf{b}^{+}+\left(\delta r_{i j}+\delta r_{i+1, j-1}\right) \mathbf{b}^{-}
\end{aligned}
$$

where $\mathbf{b}^{ \pm}$are the reciprocal lattice vectors $\mathbf{b}^{ \pm}=$ $\left( \pm 1 / 2 n_{x}, 1 / 2 n_{y}\right)$, i.e. vectors $\mathbf{b}^{ \pm}$such that $\hat{\mathbf{n}}^{ \pm} \cdot \mathbf{b}^{ \pm}=1$ and $\hat{\mathbf{n}}^{ \pm} \cdot \mathbf{b}^{\mp}=0$.

We now fix the lower boundary condition to be that the centres of all beads in the base $j=0$ have the same height and are uniformly spread horizontally: $\mathbf{x}_{i 0}=\left(2 i r n_{x}, 0\right)$, just as in the ordered case. Then (2) can be extrapolated to $j=0$ via an induction process to give the perturbed bead positions purely in terms of the $\delta r_{i j}$,

$$
\begin{aligned}
\delta \mathbf{x}_{i j}= & \left\{\delta r_{i-j, 0}+\delta r_{i j}+2 \sum_{m=1}^{j-1} \delta r_{i-(j-m), m}\right\} \mathbf{b}^{+} \\
& +\left\{\delta r_{i+j, 0}+\delta r_{i j}+2 \sum_{m=1}^{j-1} \delta r_{i+(j-m), m}\right\} \mathbf{b}^{-}
\end{aligned}
$$


Thus the position of the bead at $(i, j)$ depends on $\delta r_{i j}$, $\delta r_{i \pm 1, j-1}, \delta r_{i \pm 2, j-2}$ and so on, i.e. only on the $\delta r_{i j}$ lying along the two diagonals stretching from $(i, j)$ to the base, hereafter referred to as the 'cone.' Note that (3) ignores any horizontal boundaries, and is therefore valid for infinitely wide 'slabs' and systems with periodic boundaries at $i= \pm W$ with $W>M$, where $j=M$ denotes the maximum height of the packing.

We can now describe the manner in which the geometric disorder propagates upwards from the base. For $j \gg 1$, the expression (3) is dominated by the two sums. Thus, to good approximation, the coefficients of both the $\mathbf{b}^{+}$and $\mathbf{b}^{-}$components of $\delta \mathbf{x}_{i j}$ are the sum of $j$ independent random variables $2 \delta r_{k l}$. Each such term has a variance $4 \sigma_{\delta r}^{2}$, and so the combined variance is $\sim 4 j \sigma_{\delta r}^{2}$. Therefore the $x$ and $y$-components of $\delta \mathbf{x}_{i j}$ will both broaden like $\mathcal{O}\left(j^{1 / 2} \sigma_{\delta r}\right)$, i.e. the geometric disorder diffuses upwards in a normal (Brownian) manner. This contrasts with the non-Brownian diffusion of forces described in Sec. V.

For frictionless discs, the contact force between any two contacting beads is parallel to the line connecting the bead centres, which can be derived using (3). For instance, if the direction from the centre of bead $(i, j)$ to $(i+1, j+1)$ is denoted $\hat{\mathbf{n}}^{+}+\delta \hat{\mathbf{n}}_{i j}^{+}$, then

$$
\begin{aligned}
2 r \delta \hat{\mathbf{n}}_{i j}^{+}= & \left(\delta r_{i j}+\delta r_{i+1 j+1}\right)\left(\mathbf{b}^{+}+\mathbf{b}^{-}-\hat{\mathbf{n}}^{+}\right) \\
+ & \mathbf{b}^{-}\left\{\delta r_{i+j+2,0}-\delta r_{i+j, 0}\right. \\
& \left.+2 \sum_{m=1}^{j}\left[\delta r_{i+(j-m)+2, m}-\delta r_{i+(j-m), m}\right]\right\}
\end{aligned}
$$

This obeys $\delta \hat{\mathbf{n}}_{i j}^{+} \cdot \hat{\mathbf{n}}^{+}=0$, ensuring that $\hat{\mathbf{n}}^{+}+\delta \hat{\mathbf{n}}_{i j}^{+}$is a unit vector to first order in $\delta \hat{\mathbf{n}}_{i j}^{+}$. A similar expression applies for contacts from $(i, j)$ to $(i-1, j+1)$, which have direction $\hat{\mathbf{n}}^{-}+\delta \hat{\mathbf{n}}_{i j}^{-}$with

$$
\begin{aligned}
2 r \delta \hat{\mathbf{n}}_{i j}^{-}= & \left(\delta r_{i j}+\delta r_{i-1 j+1}\right)\left(\mathbf{b}^{+}+\mathbf{b}^{-}-\hat{\mathbf{n}}^{-}\right) \\
+ & \mathbf{b}^{+}\left\{\delta r_{i-j-2,0}-\delta r_{i-j, 0}\right. \\
& \left.+2 \sum_{m=1}^{j}\left[\delta r_{i-(j-m)-2, m}-\delta r_{i-(j-m), m}\right]\right\}
\end{aligned}
$$

\section{PROPAGATION OF FORCES FROM A SINGLE LOADED BEAD}

Consider the force propagating from a vertical load $\mathbf{F}^{\text {load }}=\left(0,-f^{\text {load }}\right)$ applied to a single bead $(k, l)$. If the packing were ordered, i.e. $\delta r_{i j} \equiv 0$ everywhere, $\mathbf{F}^{\text {load }}$ would first split equally into both of the bead's supporting contacts, and then propagate unaltered along colinear bonds to the fixed base. With disorder $\delta r_{i j} \neq 0$, there are two differences: not only is $\mathbf{F}^{\text {load }}$ shared unequally by the two contacts (which now generally have different directions), but also the propagation to the base is no longer linear but multiply branched. A qualitative description of the following analysis can be found in [23] for close-packed 3D crystals.

Let $f_{i j}^{ \pm}$denote the magnitude of the contact force from $(i, j)$ to $(i \pm 1, j+1)$, using the convention that compressive contacts have positive $f_{i j}^{ \pm}$. The force balance equation for the loaded bead $(k, l)$ is then

$$
\begin{aligned}
\mathbf{F}^{\text {load }} & +\left(\hat{\mathbf{n}}^{+}+\delta \hat{\mathbf{n}}_{k-1 l-1}^{+}\right) f_{k-1 l-1}^{+} \\
& +\left(\hat{\mathbf{n}}^{-}+\delta \hat{\mathbf{n}}_{k+1 l-1}^{-}\right) f_{k+1 l-1}^{-}=\mathbf{0}
\end{aligned}
$$

By writing $\mathbf{F}^{\text {load }}$ as $-\left(f^{\text {load }} / 2 n_{y}\right)\left(\hat{\mathbf{n}}^{+}+\hat{\mathbf{n}}^{-}\right)$, the supporting forces can be evaluated to first order in the $\delta \hat{\mathbf{n}}^{ \pm}$,

$f_{k-1 l-1}^{+}=\frac{f^{\text {load }}}{2 n_{y}}\left\{1+\frac{\phi \hat{\mathbf{n}}^{-} \cdot \delta \hat{\mathbf{n}}_{k-1 l-1}^{+}-\hat{\mathbf{n}}^{+} \cdot \delta \hat{\mathbf{n}}_{k+1 l-1}^{-}}{1-\phi^{2}}\right\}$
$f_{k+1 l-1}^{-}=\frac{f^{\text {load }}}{2 n_{y}}\left\{1+\frac{\phi \hat{\mathbf{n}}^{+} \cdot \delta \hat{\mathbf{n}}_{k+1 l-1}^{-}-\hat{\mathbf{n}}^{-} \cdot \delta \hat{\mathbf{n}}_{k-1 l-1}^{+}}{1-\phi^{2}}\right\}$

using $\phi=\hat{\mathbf{n}}^{+} \cdot \hat{\mathbf{n}}^{-}$. The range of allowed $s$ given in Sec. III means that $-\frac{1}{2}<\phi<\frac{1}{2}$, so that $1-\phi^{2}>\frac{3}{4}$ and the denominators in (7) never diverge.

Once the two contact forces $f_{k \mp 1 l-1}^{ \pm}$are known, it is necessary to calculate how they propagate to the fixed base. This is done by the same force balance equation as (6) but with $\mathbf{F}^{\text {load }}$ replaced by the corresponding incoming contact force. For instance, the force $f_{r s}^{+}$coming into a bead $(r, s)$ along the $-\left(\hat{\mathbf{n}}^{+}+\delta \hat{\mathbf{n}}_{r s}^{+}\right)$direction is balanced by the two supporting contacts $f_{r \neq 1, s-1}^{ \pm}$, which to order $\mathcal{O}\left(\delta \hat{\mathbf{n}}^{ \pm}\right)$are

$$
\begin{aligned}
& \frac{f_{r-1 s-1}^{+}}{f_{r s}^{+}}=1+\frac{\phi}{1-\phi^{2}} \hat{\mathbf{n}}^{-} \cdot\left(\delta \hat{\mathbf{n}}_{r-1 s-1}^{+}-\delta \hat{\mathbf{n}}_{r s}^{+}\right) \\
& \frac{f_{r+1 s-1}^{-}}{f_{r s}^{+}}=-\frac{1}{1-\phi^{2}} \hat{\mathbf{n}}^{-} \cdot\left(\delta \hat{\mathbf{n}}_{r-1 s-1}^{+}-\delta \hat{\mathbf{n}}_{r s}^{+}\right)
\end{aligned}
$$

The expressions for the contributions from $-f_{r s}^{-}\left(\hat{\mathbf{n}}^{-}+\right.$ $\left.\delta \hat{\mathbf{n}}_{r s}^{-}\right)$follow from symmetry. Thus the contribution of each contact force of the loaded bead $f_{k \mp 1, l-1}^{ \pm}$to any contact $f_{i j}^{ \pm}$in the system, if any, can be found by summing over all downward-propagating paths from $(k, l)$ to $(i, j)$, and applying (8) for each step. This process is simplified by the observation that the "branching" force $f_{r+1 s-1}^{-}$in (8) is $\mathcal{O}\left(\delta \hat{\mathbf{n}}^{ \pm}\right)$to leading order, and thus only paths with 0 or 1 branch point need to be considered within this first order calculation.

Without loss of generality we now consider the propagation of the $f_{k-1 l-1}^{+}$forces; the equivalent $f_{k+1 l-1}^{-}$results follow from symmetry. Suppose a contact $f_{i j}^{+}$lower 
down the packing obeys $k-i=l-j$, so that it is connected to $f_{k-1 l-1}^{+}$along a straight path with no branches. Then (8) can be applied iteratively to give

$$
\frac{f_{i j}^{+}}{f_{k-1 l-1}^{+}}=1+\frac{\phi}{1-\phi^{2}} \hat{\mathbf{n}}^{-} \cdot\left(\delta \hat{\mathbf{n}}_{i j}^{+}-\delta \hat{\mathbf{n}}_{k-1 l-1}^{+}\right)
$$

Only the $\delta \hat{\mathbf{n}}^{+}$at each end of the path contribute: the intermediate terms cancel, to first order.

Contacts $f_{i j}^{-}$with the opposite orientation may still receive a contribution from $f_{k-1 l-1}^{+}$if a path with precisely one branch point connects the two bonds. This happens if $n=\frac{1}{2}[(l-j)-(k-i)]$ obeys $1 \leq n<l-j$, in which case the branch point is at bead $(i-n, j+n)$. As the branch is $\mathcal{O}\left(\delta \hat{\mathbf{n}}^{ \pm}\right)$to leading order, only the zero'th order contributions from the remaining propagation terms are required, giving

$$
\frac{f_{i j}^{-}}{f_{k-1 l-1}^{+}}=-\frac{1}{1-\phi^{2}} \hat{\mathbf{n}}^{-} \cdot\left(\delta \hat{\mathbf{n}}_{i-n-1, j+n-1}^{+}-\delta \hat{\mathbf{n}}_{i-n, j+n}^{+}\right)
$$

All other $f_{i j}^{ \pm}$receive zero contribution. Thus the contribution to any contact force $f_{i j}^{ \pm}$due to the load $\mathbf{F}^{\text {load }}$ applied to $(k, l)$ can be found by combining the expressions (7), (9) and (10).

\section{DISTRIBUTION OF FORCES $P(F)$}

When a specified load is applied to the pile, the total contribution to any contact force $f_{i j}^{ \pm}$in the packing can be found by applying the results of Sec. IV to each loaded bead, and summing. The distribution of contact forces $P(f)$ can then be found by either averaging over all contacts with the same height, or by ensemble averaging over different packings $\left\{\delta r_{i j}\right\}$; for the loads considered here, which do not vary with horizontal distance, the final result is the same. Two alternative loadings are analysed: a surface load $\mathbf{F}^{\text {load }}=\left(0,-f^{\text {load }}\right)$ applied to all beads at $j=M$ with all other beads weightless; and a bulk load in which each bead has a weight $(0,-m g)$. Only the first case will be treated in detail here; the results of the qualitatively-similar bulk loading will be simply stated at the end.

Each contact $f_{i j}^{+}$will have contributions from the surface bead $(i+M-j, M)$ according to the unidirectional propagator (9), plus another $M-j-1$ contributions from beads in the range $(i-M+j+2, M)$ to $(i+M-j-2, M)$ which branch once according to (10). Including the initial splitting of $\mathbf{F}^{\text {load }}$ at the surface (7), the final expression for $f_{i j}^{+}$is

$$
\frac{f_{i j}^{+}}{f^{\text {load }} / 2 n_{y}}=
$$

$$
\begin{aligned}
& 1+\frac{1}{1-\phi^{2}}\left(\phi \hat{\mathbf{n}}^{-} \cdot \delta \hat{\mathbf{n}}_{i j}^{+}-\hat{\mathbf{n}}^{+} \cdot \delta \hat{\mathbf{n}}_{i+M-j+1, M-1}^{-}\right) \\
& -\frac{1}{1-\phi^{2}} \sum_{n=1}^{M-j-1} \hat{\mathbf{n}}^{+} \cdot\left(\delta \hat{\mathbf{n}}_{i+n+1, j+n-1}^{-}-\delta \hat{\mathbf{n}}_{i+n, j+n}^{-}\right)
\end{aligned}
$$

In principle, it is possible to write this expression in terms of the $\delta r_{i j}$; however, this soon becomes messy due to the proliferation of terms. Instead we now assume that $M-j \gg 1$, so that $(i, j)$ is 'sufficiently' below the surface. In this limit, the second term on the right hand side of (11) is much smaller than the sum and can therefore be dropped (recall that $\delta \hat{\mathbf{n}}_{i j}^{ \pm} \sim \mathcal{O}\left(j^{1 / 2}\right)$ ). Furthermore, the sum itself will be dominated by terms with $n=\mathcal{O}(M-j)$, so we need only consider $\delta \hat{\mathbf{n}}_{r s}^{-}$with $s=\mathcal{O}(M-j)$. This means that the sum terms in the expressions for $\delta \hat{\mathbf{n}}_{r s}^{ \pm}(4,5)$ will dominate and the remaining terms can be dropped. Combining these approximations, $f_{i j}^{+}$can be written purely in terms of combinations of $\delta r_{r s}$ like $2 \delta r_{r s}-\delta r_{r-2, s}-\delta r_{r+2, s}=\Delta_{r s}$,

$$
\begin{aligned}
\frac{f_{i j}^{+}}{f^{\text {load } / 2 n_{y}}}-1 & \approx-\frac{1}{r\left(1-\phi^{2}\right)} \sum_{n=1}^{M-j} \sum_{m=1}^{j+n} \Delta_{i-(j-m), m} \\
& \approx-\frac{1}{r\left(1-\phi^{2}\right)} \sum_{m=1}^{M} \sum_{n=\max (1, m-j)}^{M-j} \Delta_{i-(j-m), m}
\end{aligned}
$$

$$
\approx-\frac{1}{r\left(1-\phi^{2}\right)} \sum_{m=1}^{M}\left\{\begin{array}{c}
M-j: m \leq j \\
M-m: m>j
\end{array}\right\} \Delta_{i-(j-m), m}
$$

Since the mean of each $\Delta_{r s}$ is zero, we can immediately see that the mean force $\langle f\rangle=f^{\text {load }} / 2 n_{y}$ for all heights. Also, since the $\Delta_{i-(j-m), m}$ are independent for each value of $m$, then $f_{i j}^{+}$is the sum of a number of independent random variables, each of which has a weighting of no more than $\mathcal{O}(1 / M)$ of the total. Hence the central limit theorem applies, and, assuming that the variance of bead radii $\sigma_{\delta r}^{2}$ is finite, the distribution of contact forces must be Gaussian. The variance of forces $\sigma_{f}^{2}(j)$ depends on the height $j$ and can be found by standard techniques, given the weightings of the $\Delta_{i-(j-m), m}$ shown in (??). Noting that the variance of $\Delta_{r s}$ is $6 \sigma_{\delta r}^{2}$, we finally find that

$$
\frac{\sigma_{f}^{2}(z)}{\langle f\rangle^{2}} \sim \frac{2 M^{3}}{\left(1-\phi^{2}\right)^{2}}(1-z)^{2}(2 z+1)\left(\frac{\sigma_{\delta r}}{r}\right)^{2}
$$

where $z=j / M$. A graphical representation of this solution is given in Fig. 2. This should be compared with the Brownian expectation $\sigma_{j}^{2} \sim 1-z$, as seen in e.g. the $q$-model $[10,11]$. Not only is (13) superdiffusive near the surface, $\sigma_{j}^{2} \sim(1-z)^{2}$, the prefactor depends on $M$, i.e. the depth of the pile is 'felt' at the surface, no matter how large $M$ is. This is not unexpected, since the degree of geometric disorder at the surface always depends on $M$ in these near-crystalline packings; for more disordered systems, it would most likely saturate at some finite height 
and the $M$-dependence near the surface would vanish. Also note that the expression (13) becomes subdiffusive nearer the base, and ceases to broaden precisely at $j=0$. This is because the geometric disorder vanishes at the base, so that the forces no longer diffuse. To check the assumptions leading to (13), we compare it to data obtained from the adaptive network algorithm [16] in Fig. 3. The agreement is good, and appears to improve as $M$ is increased.

For a bulk loaded system, the above calculations can be repeated in a similar manner. The main difference is that the mean force now varies with depth, $\langle f\rangle_{z}=$ $M m g(1-z) / 2 n_{y}$. Nonetheless, the variance normalised by the mean force for each depth takes a similar form,

$$
\frac{\sigma_{f}^{2}(z)}{\langle f\rangle_{z}^{2}} \sim \frac{3 M^{3}}{10\left(1-\phi^{2}\right)^{2}}(1-z)^{2}(4 z+1)\left(\frac{\sigma_{\delta r}}{r}\right)^{2}
$$

The right hand side of this expression reaches a maximum at finite height $j^{*}=M / 6$, and then narrows slightly towards to the base. This is because the diffusion of forces decreases near the base, as mentioned above, but the mean force (to which the distribution is normalised) increases linearly with $1-z$, which acts to narrow the distribution. If the distribution was not normalised to $\langle f\rangle_{z}$, then it would broaden monotonically and again cease to broaden just at the base, for the same reasons as the surface loading case.

\section{A. Breakdown of the compressive bond assumption}

We can now discuss the range of validity of the assumptions made earlier. The magnitude of the displaced bead centres $\delta \mathbf{x}_{i j}$ typically vary as $\mathcal{O}\left(M^{1 / 2} \sigma_{\delta r}\right)$, which will be similar to the bead radii $r$, and hence violate the assumed contact topology, for piles of height $\mathcal{O}\left[\left(\sigma_{\delta r} / r\right)^{-2}\right]$ and greater (this assumes that the gaps between beads in the ordered packing are also $\mathcal{O}(r)$ ). However, the assumption of non-tensile contacts will be violated much sooner. For both surface and bulk loading, $\sigma_{f}$ averaged over the system has the same general form

$$
\frac{\sigma_{f}}{\langle f\rangle} \propto M^{3 / 2} \frac{\sigma_{\delta r}}{r}
$$

Thus a finite fraction of forces will become negative for packings of height $M^{*}=\mathcal{O}\left[\left(\sigma_{\delta r} / r\right)^{-2 / 3}\right]$, and the above analysis only holds for $M \ll M^{*}$. Note that this is significantly smaller than if the forces diffusion in a Brownian manner $\sim M^{1 / 2}$. Strictly speaking, we should really require that a vanishing number of contact forces are negative rather than a vanishing fraction, which would give a much lower $M^{*}$. However, this is most likely too restrictive, unless it could be shown that e.g. a single negative force initiates a cascade of rearrangements that alters the forces throughout a finite fraction of the system.

\section{RESPONSE GREEN'S FUNCTION}

The various phenomenological theories for granular stress propagation differ most markedly in the response Green's function to an isolated load [1-3,24]. This can be calculated for our system as follows: apply a load $\left(0,-f^{\text {load }}\right)$ to bead $(k, l)$, and measure the vertical component $p$ of the induced force at a contact $f_{i j}^{ \pm}$. This can be found for a given packing $\left\{\delta r_{i j}\right\}$ using the results given above. Now ensemble average over different packing realisations, i.e. uncorrelated sets of $\left\{\delta r_{i j}\right\}$ with the same variance $\sigma_{\delta r}^{2}$. Since this is a first-order calculation, the mean response will be as the ordered case, so that $\langle p\rangle_{\text {ensemble will be zero except along the cone propagating }}$ downwards from $(k, l)$. However, although the response outside this cone is strictly zero, the response inside the cone only vanishes after averaging: it will be positive or negative for each particular packing. The variance of the distribution of $p$ between different realisations can be calculated using a similar procedure to that given in Sec. V,

$$
\frac{\sigma_{\text {ensemble }}^{2}(p)}{\left(f^{\text {load }}\right)^{2}} \sim \frac{3}{2\left(1-\phi^{2}\right)^{2}}(l+j)\left(\frac{\sigma_{\delta r}}{r}\right)^{2}
$$

A schematic representation of this solution is given in Fig. 4. Interestingly, this does not decay away from the edges of the cone, as predicted by phenomenological approaches, and in fact is completely independent of the horizontal position, depending only on the combined heights $l$ and $j$.

The counterpart to ensemble averaging is coarse graining, i.e. applying a uniform pressure to a group of $N$ beads, and jointly taking $N$ and $j$ to infinity in a suitable manner to give a continuum result. However, for our packings this will just give a zero response everywhere except along the edge of the cone. This is because we have only expanded around the ordered solution to first order, which will always give the zero-th order, ordered result after any form of averaging. Extending these calculations to second order will introduce quadratic terms that do not vanish under averaging, and thus would allow a direct comparison between different types of averaging.

\section{SUMMARY AND DISCUSSION}

In summary, we have obtained analytical expressions for contact forces in isostatic packings of frictionless discs in the nearly-ordered limit. These explicitly demonstrate the anomalous broadening of the distribution of forces $P(f)$ with increasing depth, and the unusual response Green's function, whose magnitude of fluctuations under ensemble averaging depends only on the vertical coordinate. We see no reason in principle why these findings could not be tested experimentally, as long as a sufficiently monodisperse sample of smooth beads could be found. Indeed, this may be the main problem: glass bead experiments with a low-polydispersity 
of sizes placed in close-packed crystalline configurations have shown that $P(f)$ already has a broad exponential $P(f)$ at the base $[23,25]$, as opposed to the Gaussian form predicted here. This suggests that even these carefully controlled experiments are already too disordered to see the first-order results predicted here.

It is hoped that the calculations presented in this paper may lay the foundations for a more complete, analytically-tractable theory of bead packings that would complement current approaches. Thus it is worth discussing in what ways these calculations may be extended to more realistic situations. There should be little problem in going to 3 dimensions, or for considering loads at an angle to the vertical, which would allow the piles to be sheared. Perhaps more interesting would be to expand about the ordered solution to second order in the $\delta r_{i j}$, rather than just to first order as considered here. This would allow the mean force to deviate from the crystalline solution for the first time, and hence allow the nature of force propagation in granular packings to be addressed from within an analytical framework; for instance, it would be possible to see if proposed relationships between the components of the stress tensor are valid $[1,14]$. It would also show if reaching the continuum limit by ensemble averaging differs from coarse graining, as already discussed in Sec. VI.

However, extending this analysis to frictional beads is likely to be more troublesome. The marginal rigidity state for beads with infinite friction has $Z=3$ in 2 dimensions, so that sequentially deposited beads will make either 1 or 2 contacts on the surface - in the case of 1 contact, it is not possible to determine the rest position of the incoming bead. Thus groups of beads must be added simultaneously, so that the final geometry will depend on incoming bead trajectories and their material properties. Also, with $Z=3$ it is not possible to uniquely decompose the load applied to any given bead along paths connecting it to the base, for either the normal or tangential contact forces. Instead loops, and also paths leading to the surface, will inevitably arise. This non-unique decomposition will also be a problem for frictionless non-spherical beads, for which $Z=6$ in 2 dimensions. It is not clear how these problems may be surmounted without resorting to ad hoc assumptions or simplifications.

\section{ACKNOWLEDGMENTS}

The author would like to thank Mike Cates and Jacco Snoeijer for correspondence relating to this work. This research was partly funded through a European Community Marie Curie Fellowship.
[1] M.E. Cates, J.P. Wittmer, J.-P. Bouchaud and P. Claudin 1996 Phil. Trans. Roy. Soc. Lond. A 356 2535; also cond-mat/9803266

[2] J.P. Bouchaud to appear in Proceedings of Les Houches Summer School 2002; cond-mat/0211196

[3] M. Otto, J.-P. Bouchaud, P. Claudin and J.E.S. Socolar 2003 Phys. Rev. E 67031302

[4] E. Guyon, S. Roux, A. Hansen, D. Bideau, J.-P. Troadec and H. Crapo 1990 Rep. Prog. Phys. 53373

[5] R.C. Ball and R. Blumenfeld 2002 Phys. Rev. Lett. 88 115505

[6] S.F. Edwards and D.V. Grinev 1999 Phys. Rev. Lett. 82 5397

[7] C. Gay and R. da Silveira preprint cond-mat/0208155

[8] R. da Silveira, G. Vidalenc and C. Gay preprint condmat/0208214

[9] S.N. Coppersmith et al. 1996 Phys. Rev. E 534673

[10] J.H. Snoeijer and J.M.J. van Leeuwen 2002 J. Stat. Phys. 109449

[11] J.H. Snoeijer and J.M.J. van Leeuwen 2002 Phys. Rev. E 65051306

[12] J.-P. Bouchaud, P. Claudin, D. Levine and M. Otto 2001 Euro. Phys. J. E 4451

[13] J.E.S. Socolar, D.G. Schaeffer and P. Claudin 2002 Euro. Phys. J. E 7353

[14] A.V. Tkachenko and T.A. Witten 1999 Phys. Rev. E 60 687

[15] D.A. Head, A.V. Tkachenko and T.A. Witten 2001 Euro. Phys. J. E 699

[16] A.V. Tkachenko and T.A. Witten 2000 Phys. Rev. E 62 2510

[17] J.-N. Roux 2000 Phys. Rev. E 616802

[18] S. Ouaguenouni and J.-N. Roux 1997 Euro. Phys. Lett. 39117

[19] L. Breton, P. Claudin, É. Clément and J.-D. Zucker 2002 Euro. Phys. Lett. 60813

[20] C.F. Moukarzel 1998 Phys. Rev. Lett. 811634

[21] C.F. Moukarzel 2002 J. Phys. Cond. Mat. 142379

[22] Formally: start from the ordered packing, for which every contact force is known to be finite and compressive. Now increase the polydispersity of bead radii continuously from zero. Since the contact forces will also vary continuously (for systems of finite size), they must remain compressive for some finite range of polydispersity.

[23] N.W. Mueggenburg, H.M. Jaeger and S.R. Nagel 2002 Phys. Rev. E 66031304

[24] J. Geng, G. Reydellet, E. Clément and R.P. Behringer preprint cond-mat/0211031

[25] D.L. Blair, N.W. Mueggenburg, A.H. Marshall, H.M. Jaeger and S.R. Nagel 2001 Phys. Rev. E 63041304 


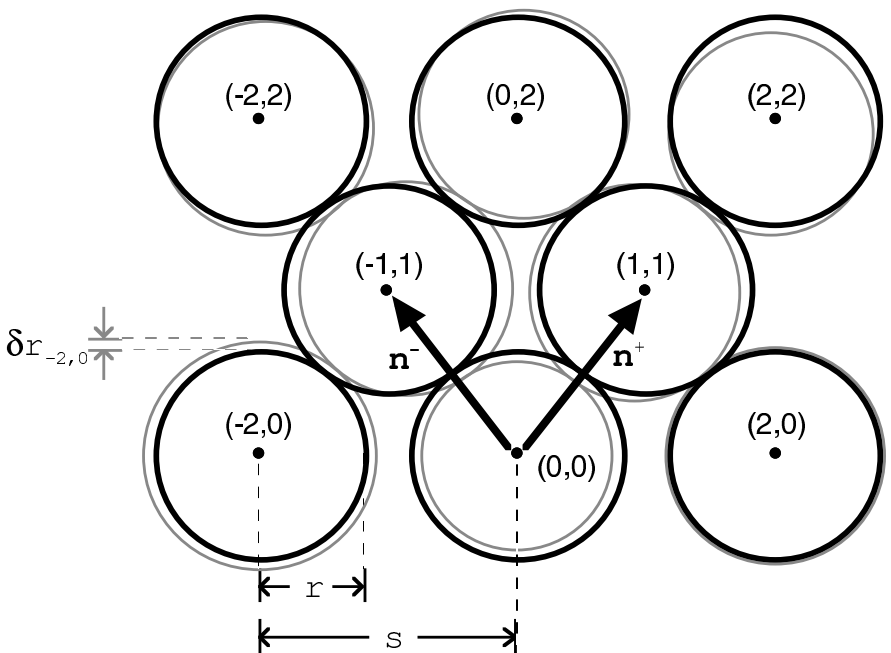

FIG. 1. An ordered packing of discs of radius $r$ and horizontal separation $s$ (thick black circles). The lattice indices $(i, j)$ and the lattice base vectors $\hat{\mathbf{n}}^{ \pm}$are also shown. A particular example of a disordered packing, in which the beads have radii $r+\delta r_{i j}$, is superimposed in grey.

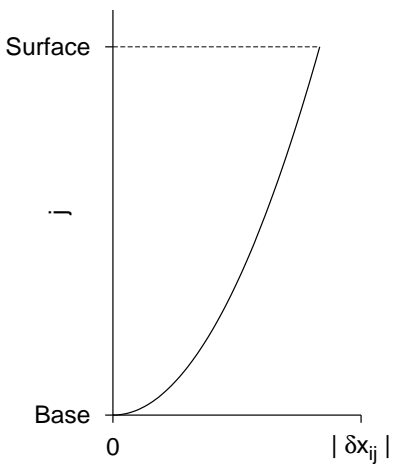

(a)

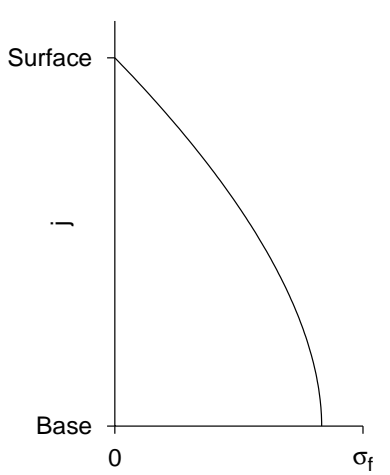

(b)
FIG. 2. Graphical representation of (a) the broadening of the geometric disorder with height $j$, and (b) the broadening of the force distribution with distance from the surface, as given by the surface-loaded solution (13).

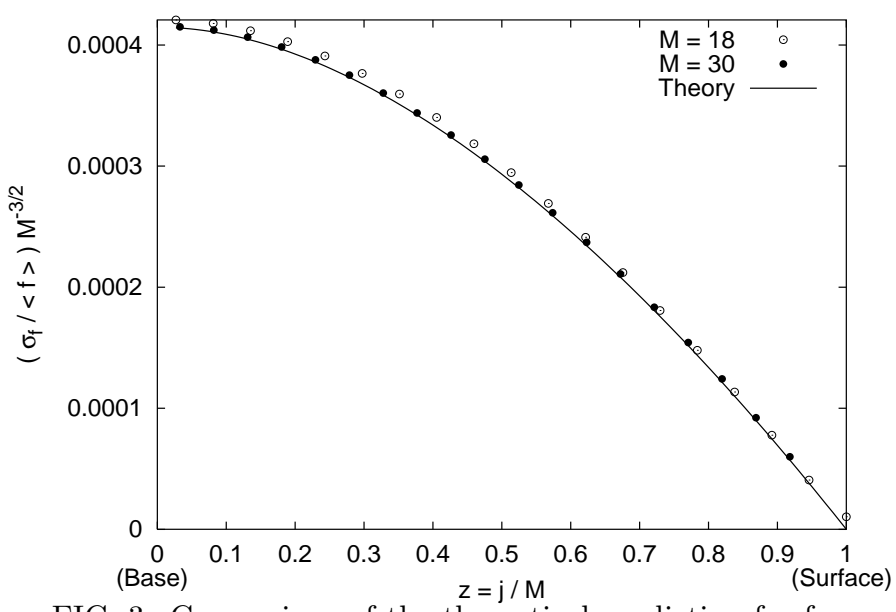

FIG. 3. Comparison of the theoretical prediction for force diffusion for a surface-loaded system (13) with corresponding simulation data. The normalised standard deviation of forces $\sigma_{f} /\langle f\rangle$ has been scaled by $M^{3 / 2}$ so that, according to (13), the curve will be $M$-independent when plotted against the relative height $z=j / M$. The two pile heights used in the simulations are given in the key, and appear to confirm this prediction. The parameters for both theory and simulations are $\left(\sigma_{\delta r} / r\right)^{2}=10^{-6} / 12, r=1$ and $s=3$.

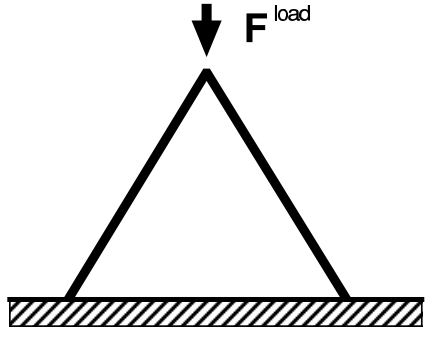

(a)

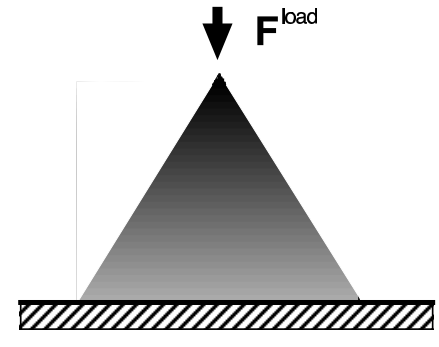

(b)
FIG. 4. Schematic representation of the Green's response solution (16) to a vertical load $\mathbf{F}^{\text {load }}$. (a) The mean response is identical to the corresponding ordered system, in which forces propagate along lines of colinear bonds forming a 'cone.' (b) The magnitude of the fluctuations under ensemble-averaging, with darker grey corresponding to a greater variance. The variance is zero outside the cone, depends only on height inside the cone, and is twice as large near the apex as near the base (fluctuations on the cone itself are not shown). 